\section{Associação dos padrões alimentares com obesidade geral e abdominal em mulheres residentes no Sul do Brasil}

\author{
Association between dietary patterns and body \\ mass index and waist circumference in women \\ living in Southern Brazil
}

\footnotetext{
${ }_{1}^{1}$ Programa de Pós-graduação em Saúde Coletiva, Universidade do Vale do Rio dos Sinos, São Leopoldo, Brasil.

Correspondência M. T. A. Olinto Programa de Pós-graduação em Saúde Coletiva Universidade do Vale do Rio dos Sinos. Av. Unisinos 950 São Leopoldo, RS 93022-000, Brasil. mtolinto@unisinos.br
}

\begin{abstract}
This study focused on the association between dietary patterns and obesity. A cross-sectional population-based study was performed in 1,026 adult women from Southern Brazil. Waist circumference (WC), height, and weight were measured according to a standardized protocol, and body mass index (BMI) was calculated. Obesity was defined as $B M I \geq 30 \mathrm{~kg} / \mathrm{m}^{2}$ and $W C \geq 88 \mathrm{~cm}$. Principal components analysis was used to identify dietary patterns. Multivariate analysis used a Poisson regression model to estimate prevalence ratios and respective confidence intervals. After controlling for possible confounders, low consumption of "vegetables" protected against increased BMI (PR = 0.64; 95\%CI: 0.47-0.86; $p=$ 0.004), while low consumption of "nuts/oilseeds" protected against increased WC $(P R=0.93$; 95\%CI: 0.89-0.98; $p=0.008)$. Low consumption of "fruits" was a risk factor for high BMI (PR = 2.18; 95\%CI: 1.35-3.53; $p=0.001$ ). The study indicates the complexity of the association between dietary patterns and obesity. New studies are needed to further clarify the subject.
\end{abstract}

Feeding Behavior; Obesity; Abdominal Fat; Women

\author{
Gabriela Perozzo ${ }^{1}$ \\ Maria Teresa Anselmo Olinto 1 \\ Juvenal Soares Dias-da-Costa ${ }^{1}$ \\ Ruth Liane Henn 1 \\ Jorge Sarriera 1 \\ Marcos Pascoal Pattussi 1
}

\section{Introdução}

A obesidade tem sido apontada como problema de saúde pública desde meados da década de 80 1. A partir de então, em diversas regiões do mundo, intensificaram-se as investigações sobre prevalências de obesidade e os fatores a ela associados. No Brasil, na década de 70, o Estudo Nacional da Despesa Familiar (ENDEF 1974-1975) 2 apontou prevalências de 18,6\% de excesso de peso (índice de massa corporal - IMC $\geq 25 \mathrm{~kg} / \mathrm{m}^{2}$ ) entre os homens e de $28,6 \%$ entre as mulheres, sendo $2,8 \%$ e $7,8 \%$ de obesidade (IMC $\geq 30 \mathrm{~kg} / \mathrm{m}^{2}$ ), respectivamente. Atualmente, de acordo com dados da Pesquisa de Orçamentos Familiares (POF 2002-2003) 3, o país apresenta prevalência de $40,6 \%$ de excesso de peso em adultos, sendo $8,9 \%$ de homens e $13,1 \%$ de mulheres obesos.

No Sul do Brasil, estudos recentes indicam prevalência de excesso de peso de $53 \%$, sendo $33,7 \%$ de sobrepeso e $19,4 \%$ de obesidade ${ }^{4}$. Na mesma região, as prevalências de obesidade abdominal são de $62 \%$ nas mulheres e de $37 \%$ nos homens ${ }^{5}$.

Além dos determinantes sócio-econômicos e demográficos, também têm sido investigados alguns comportamentos alimentares como fator de risco para sobrepeso e obesidade. O hábito de continuar comendo após sentir-se saciado ${ }^{6}$, o menor número de refeições diárias 7 e o maior número de refeições realizadas fora de 
casa 8 estiveram associados ao aumento do peso corporal. Por outro lado, a inclusão do café da manhã apresenta-se como fator protetor 7 .

A gênese da obesidade também tem sido avaliada no âmbito da identificação do papel de nutrientes, como gorduras, carboidratos e fibras, além do índice glicêmico de alimentos, para que uma adequada prevenção possa ser realizada. No entanto, a etiologia nutricional desta patologia ainda permanece controversa, especialmente com relação ao papel da gordura da dieta e dos carboidratos 9,10,11,12,13.

A dieta também pode influenciar o desenvolvimento da adiposidade abdominal, mas poucos estudos têm examinado tal relação. Uma dieta rica em fibras 14,15,16,17 e com alimentos de baixo índice glicêmico 15,17 pode resultar em menores níveis de adiposidade central. Já a elevada ingestão de gordura trans 16 e de carboidratos simples 14,18 tem sido associada ao ganho na medida da circunferência da cintura (CC).

Grande parte desses estudos tem analisado a associação entre risco de doenças crônicas e dieta, sendo esta avaliada pela ingestão de nutrientes ou de alimentos. Porém, as pessoas não os ingerem de forma isolada, mas em refeições compostas de uma variedade de alimentos, com complexas combinações de nutrientes que podem ser observadas somente quando padrões alimentares são considerados 19 .

Conceitualmente, padrões alimentares representam um retrato geral do consumo de alimentos e de nutrientes, caracterizados com base no hábito de ingestão usual. Dessa maneira, a análise de padrões poderia predizer melhor o risco de doenças do que a de nutrientes ou de alimentos isolados, visto que o efeito cumulativo de múltiplos nutrientes incluídos em um padrão alimentar seria melhor detectado 19.

A associação de padrões alimentares com excesso de peso tem sido objeto de interesse de vários estudos. Alguns deles apontam associação positiva de padrões alimentares com obesidade global e central 17,20,21,22,23,24,25,26. Em outros estudos, entretanto, essa relação não é clara 27,28,29, sobretudo quando se observa a consistência dessa associação ${ }^{30}$.

O presente estudo tem como objetivo investigar a associação de padrões alimentares encontrados em mulheres adultas, com obesidade geral e abdominal.

\section{Materiais e métodos}

Foi realizado um estudo transversal de base populacional com amostra representativa de 1.026 mulheres de 20 a 60 anos residentes na zona ur- bana de São Leopoldo, Região do Vale do Rio dos Sinos, Rio Grande do Sul, Brasil.

O tamanho da amostra foi calculado mediante diferentes desfechos a serem investigados no estudo. Escolheu-se o maior tamanho de amostra para encontrar uma razão de risco de 2,0, com nível de $95 \%$ de confiança, poder estatístico de $80 \%$ e mantida a razão de não expostos:expostos de 1:3 para a variável classe econômica. Considerando possíveis perdas/recusas durante o trabalho de campo e controle de fatores de confusão na análise dos dados, a amostra foi acrescida em 25\%, sendo necessárias 1.358 mulheres.

Baseando-se em dados do Instituto Brasileiro de Geografia e Estatística (IBGE) do Censo Demográfico 2000 (http://www.ibge.gov.br), estimou-se, em média, 3,35 pessoas/domicílio no município e $28,2 \%$ da população constituída de mulheres na faixa etária de interesse. A seleção da amostra foi realizada em múltiplos-estágios por intermédio dos setores censitários, quarteirões e domicílios. Todas as mulheres de 20 a 60 anos residentes nos domicílios sorteados foram incluídas no estudo. A coleta de dados ocorreu entre março e novembro do ano de 2003. Maiores detalhes são encontrados em outras publicações 31,32 .

As informações sócio-econômicas, demográficas e comportamentais foram coletadas por questionário padronizado, pré-codificado e prétestado, aplicado por entrevistadores submetidos a um programa de treinamento. Como variáveis sócio-econômicas utilizaram-se: (a) classe econômica - classificada conforme os critérios da Associação Brasileira de Empresas de Pesquisa (ABEP) 33 e categorizada em "A+B", "C", "D+E"; (b) renda familiar per capita - obtida pela soma da renda de cada integrante da família no último mês, dividida pelo número de membros na família e categorizada em salários mínimos per capita; (c) escolaridade - coletada em anos completos de estudo e sendo categorizada em tercis; e (d) situação de trabalho - avaliada no momento da entrevista. Como variáveis demográficas utilizaram-se: (a) idade - coletada em anos completos no momento da entrevista e categorizada tanto em faixas etárias de dez anos, para descrição da amostra, como em uma variável dicotômica com o ponto de corte de 35 anos; (b) estado civil - informado pela entrevistada e classificado em "com" ou "sem" companheiro; e (c) cor da pele - observada pelo entrevistador e categorizada em branca e não branca. Como variáveis comportamentais investigaram-se: (a) atividade física - classificada em moderadamente ativo ou não, sendo consideradas moderadamente ativas as mulheres que informaram praticar atividade física no lazer de forma média ou forte, três vezes 
ou mais por semana 32; e (b) fumo - sendo as mulheres classificadas como fumante, ex-fumante e nunca fumou.

Os padrões alimentares utilizados foram obtidos, nesta amostra, por um Questionário de Freqüência Alimentar (QFA) semiquantitativo com setenta alimentos - em outra publicação apresenta-se detalhadamente a identificação dos padrões e sua denominação ${ }^{32}$. Os cinco padrões alimentares explicaram $28,2 \%$ da variância, e alimentos com valores de saturação superiores a 0,30 foram considerados como componentes para o padrão alimentar. Para facilitar a compreensão da associação do padrão alimentar com obesidade, no presente artigo, os padrões alimentares identificados em Alves et al. 31 foram renomeados de acordo com as maiores cargas de saturação dos alimentos no fator, ficando assim denominados: PA-vegetais (repolho, couve, couve-flor, couve de bruxelas, abóbora, cenoura, laranja, brócolis, pepino, beterraba, tomate, vagem, banana, mamão, maçã, bergamota, outros vegetais verdes e biscoito salgado); PA-frutas (melão, melancia, manga, pêra, pêssego, caqui, uva, limão, maracujá, abacaxi, sorvete, abacate, goiaba, kiwi, fígado, suco natural e peixes); PAnozes/oleaginosas (amêndoa, avelã, nozes, castanha, ameixa seca, uva passa, massa integral, aveia, farelo de trigo, açúcar mascavo, mel, pão de centeio, pão integral, soja, vinho tinto e arroz integral); PA-pão/aipim/batata doce (pão caseiro, aipim, batata doce, milho, feijão, lentilha, açúcar, banha, leite integral, batata inglesa, nata, massa e carne de porco); e PA-chocolate/doces (chocolate, balas, sobremesas, doces, creme de leite, presunto, mortadela, salame, copa, maionese industrializada, queijo, frituras, fast food, biscoito doce, cuca e bolo).

Para o presente estudo, cada um dos padrões alimentares foi categorizado em variável dicotômica, de acordo com os escores de cada padrão (variação de 10 a 58), sendo considerada a categoria de alto consumo para o quintil superior da distribuição e baixo consumo para os escores inferiores.

Os desfechos deste estudo são obesidade geral e abdominal. A obesidade geral foi avaliada pelo IMC, que caracteriza distribuição generalizada de gordura, e a obesidade abdominal, pela medida da CC, relacionada com o acúmulo central de adiposidade.

Para o cálculo do IMC (peso em kg/altura em $\mathrm{m}^{2}$ ), foram coletadas as medidas de peso e estatura, seguindo as recomendações da Organização Mundial da Saúde (OMS) 1. O peso foi aferido com uma balança mecânica, marca Sunrise (Metalúrgica Promesul, São Leopoldo, Brasil), com precisão de 100g. Foram coletadas duas medidas e a seguir realizada a média dos valores, sendo esta utilizada para o cálculo de IMC. Para a medida da estatura, utilizou-se estadiômetro da marca Seca Body Meter (Seca, Hamburgo, Alemanha), com precisão de $1 \mathrm{~mm}$. A leitura foi realizada em apnéia respiratória. Foram coletadas duas medidas e calculada a média dos valores, a qual foi utilizada no cálculo do IMC.

A variável IMC foi categorizada de duas maneiras. Para descrever a amostra, as mulheres foram classificadas em IMC normal (IMC < $\left.25 \mathrm{~kg} / \mathrm{m}^{2}\right)$, sobrepeso $\left(25 \mathrm{~kg} / \mathrm{m}^{2} \leq \mathrm{IMC}<30 \mathrm{~kg} / \mathrm{m}^{2}\right)$ e obesidade (IMC $\geq 30 \mathrm{~kg} / \mathrm{m}^{2}$ ) 1 . Para as análises brutas e ajustadas o IMC foi categorizado em variável dicotômica: mulheres não obesas (IMC < $30 \mathrm{~kg} / \mathrm{m}^{2}$ ) e obesas (IMC $\geq 30 \mathrm{~kg} / \mathrm{m}^{2}$ ).

Para medir a circunferência da cintura, utilizou-se fita métrica Sanny (American Medical do Brasil Ltda., São Bernardo do Campo, Brasil), com precisão de $1 \mathrm{~mm}$, no ponto de menor diâmetro abaixo da última costela, entre o último arco costal e a crista ilíaca 1 . A medida foi coletada duas vezes e, posteriormente, a média dos valores encontrados foi calculada, sendo este o valor utilizado para o estudo. Para descrever a amostra, a variável CC foi categorizada de acordo com os níveis de intervenção propostos por Lean et al. 34: normal $(\mathrm{CC}<80 \mathrm{~cm})$, nível I $(80 \mathrm{~cm} \leq \mathrm{CC}<88 \mathrm{~cm})$ e nível II (CC $\geq 88 \mathrm{~cm}$ - obesidade abdominal), enquanto as análises bivariadas e multivariadas foram realizadas com a variável dicotomizada: $\mathrm{CC}<88 \mathrm{~cm}$ e $\mathrm{CC} \geq 88 \mathrm{~cm}$.

A entrada dos dados foi realizada por meio do programa Epi Info 6.0 (Centers for Disease Control and Prevention, Atlanta, Estados Unidos), em dupla digitação para verificação de inconsistências. As análises foram realizadas no SPSS 13.0 para Windows (SPSS Inc., Chicago, Estados Unidos) e Stata 7.0 (Stata Corp., College Station, Estados Unidos).

O efeito do delineamento para o desfecho obesidade abdominal foi de 1,115 e, portanto, as análises levaram em consideração o desenho amostral mediante o conjunto de comandos svy do Stata. Para o desfecho obesidade (IMC), o efeito de delineamento foi de 0,982 , logo, as análises não foram realizadas com correção para o desenho do estudo.

As etapas da análise incluíram: freqüência simples para descrição da amostra; análise bruta das variáveis sócio-econômicas, demográficas, comportamentais e padrões alimentares com os desfechos obesidade geral e abdominal (teste do qui-quadrado, razões de prevalência - RP e intervalos de 95\% de confiança - IC95\%); análise estratificada e análise ajustada.

A análise estratificada foi realizada para os seguintes estratos: idade (20-35 anos e 36-60 
anos), escolaridade (em tercis: $0-5,6-10,11$ ou mais anos de estudo) e classe econômica ("A+B", "C", "D+E"). A análise multivariada para investigar associação das variáveis antropométricas com os padrões alimentares, controlando para potenciais fatores de confusão (variáveis sócioeconômicas, demográficas e comportamentais), foi realizada pela regressão de Poisson com variância robusta. Essa análise seguiu uma abordagem hierarquizada de determinação 35 (Figura 1). No nível distal estão as variáveis sócio-econômicas e demográficas, no intermediário, as comportamentais e no nível proximal encontra-se a associação de interesse "padrão alimentar e obesidade", que é influenciada e dependente dos níveis distal e intermediário. Em razão da possibilidade de causalidade reversa entre padrão alimentar e obesidade, estão sendo apresentados a exposição (padrões alimentares) e o desfecho (obesidade) no mesmo nível do modelo. Somente as variáveis que apresentaram valor de $\mathrm{p}<0,2$ na análise bruta foram levadas para a análise ajustada. Para ser considerado fator de confusão para o próximo nível do modelo, a variável deve- ria apresentar valor de $\mathrm{p}<0,1$ quando ajustada pelas variáveis do seu nível. Ao final, foram consideradas fatores associados à obesidade geral ou à obesidade abdominal as variáveis com nível de significância menor que $5 \%$.

Este estudo foi aprovado pelo Comitê de Ética e Pesquisa da Universidade do Vale do Rio dos Sinos. Após esclarecimentos sobre a pesquisa, as mulheres que aceitaram participar do estudo assinaram o Termo de Consentimento Livre e PréInformado.

\section{Resultados}

Entre as 1.084 mulheres de 20 e 60 anos detectadas pelo estudo, foram verificados $5,4 \%$ de perdas e recusas, de forma que 1.026 mulheres foram incluídas na análise. Na Tabela 1, observa-se que mais da metade da amostra tinha idade entre 20 e 39 anos, com predomínio de mulheres de cor da pele branca e de mulheres que viviam com companheiro. Apenas $4,6 \%$ da amostra tinham cor da pele negra. Quanto aos fatores sócio-eco-

Figura 1

Modelo hierarquizado multivariado de análise da associação de padrões alimentares com obesidade geral e abdominal.

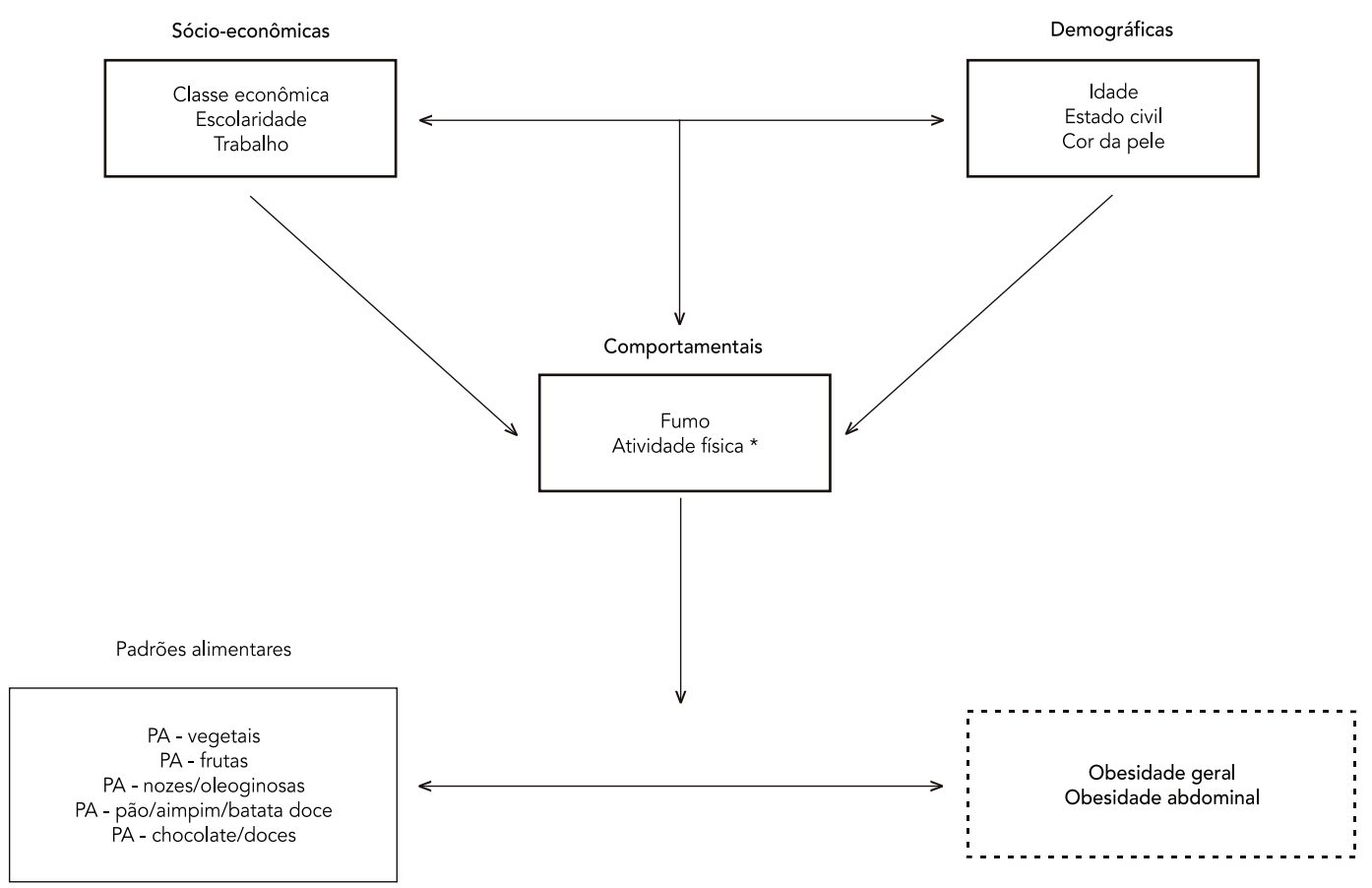

* Esta variável está presente apenas nas análises multivariadas para o PA-vegetais, o PA-frutas e o PA-nozes/oleaginosas. 
nômicos, cerca de $37 \%$ das mulheres tinham renda mensal per capita de até um salário mínimo, e mais de $1 / 4$ pertencia às classes econômicas “D+E”. Em relação à escolaridade, 1/3 da amostra apresentava menos de cinco anos de estudo e $42,4 \%$ delas não estavam trabalhando no momento da pesquisa. Quanto às características comportamentais, a maioria $(68,5 \%)$ das mulheres não praticava atividade física regularmente e $22,5 \%$ eram fumantes.

Considerando as formas mais graves de obesidade, a maior prevalência foi observada para a obesidade abdominal, em que 23,3\% (IC95\%: 20,72-26,06) das mulheres apresentaram CC $\geq$ $88 \mathrm{~cm}$ (nível II), em comparação à prevalência de 18\% (IC95\%: 15,66-20,53) de mulheres obesas (IMC $\geq 30 \mathrm{~kg} / \mathrm{m}^{2}$ ) (Tabela 1).

A Tabela 2 apresenta as razões de prevalências brutas para obesidade segundo as características sócio-econômicas, demográficas, comportamentais e os padrões alimentares. As variáveis sócioeconômicas e demográficas que apresentaram associação com a obesidade foram as mesmas para obesidade geral (IMC) e para adiposidade abdominal (CC): classe econômica, escolaridade, situação de trabalho, idade e estado civil. Quanto à associação dos padrões alimentares com os dois tipos de obesidade, baixo consumo de frutas (PA-frutas) parece indicar risco tanto para obesidade geral como abdominal. Por outro lado, o baixo consumo de PA-chocolates/doces também aponta para risco de ocorrência desses desfechos. Nas análises brutas, observam-se associações dos padrões alimentares com obesidade abdominal com intervalos de confiança limítrofes para o efeito. Observa-se, ainda, o efeito protetor limítrofe do baixo consumo de PA-vegetais sobre a obesidade geral e do baixo consumo de PA-nozes/oleaginosas sobre a adiposidade abdominal.

$\mathrm{Na}$ análise ajustada (Tabela 3), a proteção para obesidade geral e abdominal mantém-se à medida que aumenta a escolaridade. Considerando as mulheres que trabalham, existe uma tendência de proteção para a obesidade geral $(\mathrm{RP}=0,76$; IC95\%: 0,58-1,00; $\mathrm{p}=0,055)$ e efeito protetor significativo para obesidade abdominal $(\mathrm{RP}=0,92$; IC95\%: 0,88-0,96; $\mathrm{p}<0,001)$. O risco aumentado para ambas as medidas de obesidade mantém-se para as mulheres com idade acima de 35 anos.

Ainda na Tabela 3, observa-se que, após controlar para fatores de confusão (idade, estado civil, escolaridade e variáveis sócio-econômicas), o baixo consumo do PA-frutas manteve-se como risco para a obesidade. Por outro lado, a associação do PA-chocolate/doces, tanto com obesidade geral como com obesidade abdominal, desaparece.
Tabela 1

Características sócio-econômicas, demográficas, comportamentais e antropométricas de mulheres adultas do Município de São Leopoldo, Rio Grande do Sul, Brasil, 2003 ( $N=1.026$ ).

\begin{tabular}{|c|c|c|}
\hline Características & $\mathbf{n}$ & $\%$ \\
\hline \multicolumn{3}{|l|}{ Demográficas } \\
\hline \multicolumn{3}{|l|}{ Idade (anos) } \\
\hline $20-29$ & 283 & 27,6 \\
\hline $30-39$ & 255 & 24,9 \\
\hline $40-49$ & 302 & 29,4 \\
\hline $50-60$ & 186 & 18,1 \\
\hline \multicolumn{3}{|l|}{ Estado civil } \\
\hline Com companheiro & 659 & 64,2 \\
\hline Sem companheiro & 367 & 35,8 \\
\hline \multicolumn{3}{|l|}{ Cor da pele } \\
\hline Branca & 860 & 83,8 \\
\hline Não branca & 166 & 16,2 \\
\hline \multicolumn{3}{|l|}{ Sócio-econômicas } \\
\hline \multicolumn{3}{|l|}{ Classe econômica * } \\
\hline "D+E" & 268 & 26,2 \\
\hline "C" & 404 & 39,5 \\
\hline$" A+B "$ & 351 & 34,3 \\
\hline \multicolumn{3}{|c|}{ Renda familiar per capita (salários míninos) * } \\
\hline $0-1$ & 373 & 36,8 \\
\hline $1,01-3$ & 427 & 42,1 \\
\hline $3,01-6$ & 141 & 13,9 \\
\hline 6,01 ou mais & 73 & 7,2 \\
\hline \multicolumn{3}{|c|}{ Escolaridade (anos de estudo) * } \\
\hline $0-5$ & 338 & 33,5 \\
\hline $6-10$ & 268 & 26,6 \\
\hline 11 ou mais & 402 & 39,9 \\
\hline \multicolumn{3}{|l|}{ Trabalho } \\
\hline Não & 435 & 42,4 \\
\hline Sim & 591 & 57,7 \\
\hline \multicolumn{3}{|l|}{ Comportamentais } \\
\hline \multicolumn{3}{|l|}{ Fumo } \\
\hline Nunca fumou & 606 & 59,1 \\
\hline Ex-fumante & 189 & 18,4 \\
\hline Fumante & 231 & 22,5 \\
\hline \multicolumn{3}{|l|}{ Moderadamente ativo } \\
\hline Sim & 323 & 31,5 \\
\hline Não & 703 & 68,5 \\
\hline \multicolumn{3}{|l|}{ Antropométricas } \\
\hline \multicolumn{3}{|c|}{ Índice de massa corporal $\left(\mathrm{kg} / \mathrm{m}^{2}\right)$ ** } \\
\hline$<18,5$ & 15 & 1,5 \\
\hline $18,5-24,9$ & 490 & 49,2 \\
\hline $25,0-29,9$ & 312 & 31,3 \\
\hline$\geq 30$ & 179 & 18,0 \\
\hline \multicolumn{3}{|c|}{ Circunferência da cintura $(\mathrm{cm})$ ** } \\
\hline Normal $(<80)$ & 536 & 53,8 \\
\hline Nível I (80-87) & 228 & 22,9 \\
\hline Nível II ( $\geq 88)$ & 232 & 23,3 \\
\hline
\end{tabular}

* Não foi possível coletar a informação sobre classe econômica em três mulheres, sobre a renda em 12 mulheres e, em relação à escolaridade, foram 18 mulheres;

** Não foi possível coletar a medida de peso e de circunferência da cintura em trinta muIheres. 
Razões de prevalência (RP) brutas e intervalos de 95\% de confiança (IC95\%) para obesidade geral (índice de massa corporal - IMC) e abdominal (circunferência de cintura - CC) segundo características demográficas, sócio-econômicas, comportamentais e elevado consumo dos padrões alimentares * em mulheres adultas de São Leopoldo, Rio Grande do Sul, Brasil, 2003 $(\mathrm{N}=1.026)$.

\begin{tabular}{|c|c|c|c|c|c|c|}
\hline \multirow[t]{2}{*}{ Variáveis } & \multicolumn{3}{|c|}{ IMC } & \multicolumn{3}{|c|}{$\mathrm{CC}$} \\
\hline & $\mathrm{RP}$ & IC95\% & Valor de $p$ & RP & IC95\% & Valor de $p$ \\
\hline \multicolumn{7}{|l|}{ Sócio-econômicas } \\
\hline \multicolumn{7}{|l|}{ Classe econômica } \\
\hline "D+E" & 1,00 & & 0,013 & 1,00 & & 0,014 \\
\hline "C" & 0,77 & $0,54-1,05$ & & 0,92 & $0,88-0,97$ & \\
\hline$" A+B "$ & 0,59 & $0,42-0,84$ & & 0,92 & $0,87-0,98$ & \\
\hline \multicolumn{7}{|l|}{ Escolaridade (anos) } \\
\hline $0-5$ & 1,00 & & $<0,001$ & 1,00 & & $<0,001$ \\
\hline $6-10$ & 0,65 & $0,47-0,90$ & & 0,88 & $0,83-0,93$ & \\
\hline 11 ou mais & 0,42 & $0,30-0,58$ & & 0,85 & $0,81-0,89$ & \\
\hline \multicolumn{7}{|l|}{ Trabalho } \\
\hline Não & 1,00 & & 0,002 & 1,00 & & $<0,001$ \\
\hline Sim & 0,65 & $0,50-0,85$ & & 0,89 & $0,85-0,93$ & \\
\hline \multicolumn{7}{|l|}{ Idade (anos) } \\
\hline $20-35$ & 1,00 & & $<0,001$ & 1,00 & & $<0,001$ \\
\hline $36-60$ & 2,03 & $1,48-2,78$ & & 1,14 & $1,09-1,19$ & \\
\hline \multicolumn{7}{|l|}{ Demográficas } \\
\hline \multicolumn{7}{|l|}{ Estado civil } \\
\hline Com companheiro & 1,00 & & 0,031 & 1,00 & & 0,002 \\
\hline Sem companheiro & 0,72 & $0,53-0,97$ & & 0,93 & $0,89-0,97$ & \\
\hline \multicolumn{7}{|l|}{ Cor da pele } \\
\hline Branca & 1,00 & & 0,220 & 1,00 & & 0,433 \\
\hline Não branca & 1,23 & $0,88-1,71$ & & 1,02 & $0,96-1,07$ & \\
\hline \multicolumn{7}{|l|}{ Comportamentais } \\
\hline \multicolumn{7}{|l|}{ Fumo } \\
\hline Nunca fumou & 1,00 & & 0,630 & 1,00 & & 0,623 \\
\hline Ex-fumante & 1,12 & $0,80-1,57$ & & 1,02 & $0,97-1,08$ & \\
\hline Fuma & 0,91 & $0,65-1,29$ & & 1,00 & $0,95-1,06$ & \\
\hline \multicolumn{7}{|l|}{ Atividade física } \\
\hline Sim & 1,00 & & 0,632 & 1,00 & & 0,329 \\
\hline Não & 1,07 & $0,80-1,43$ & & 1,02 & $0,97-1,07$ & \\
\hline \multicolumn{7}{|l|}{ Padrões alimentares } \\
\hline \multicolumn{7}{|l|}{ PA-vegetais } \\
\hline Elevado consumo & 1,00 & & 0,049 & 1,00 & & 0,374 \\
\hline Baixo consumo & 0,73 & $0,54-0,99$ & & 0,97 & $0,93-1,02$ & \\
\hline \multicolumn{7}{|l|}{ PA-frutas } \\
\hline Elevado consumo & 1,00 & & 0,002 & 1,00 & & 0,023 \\
\hline Baixo consumo & 2,06 & $1,31-3,23$ & & 1,06 & $1,00-1,12$ & \\
\hline \multicolumn{7}{|l|}{ PA-nozes/oleaginosas } \\
\hline Elevado consumo & 1,00 & & 0,951 & 1,00 & & 0,006 \\
\hline Baixo consumo & 0,98 & $0,70-1,38$ & & 0,93 & $0,89-0,98$ & \\
\hline \multicolumn{7}{|c|}{ PA-pão/aipim/batata doce } \\
\hline Elevado consumo & 1,00 & & 0,395 & 1,00 & & 0,166 \\
\hline Baixo consumo & 0,86 & $0,62-1,20$ & & 0,96 & $0,91-1,01$ & \\
\hline \multicolumn{7}{|l|}{ PA-chocolate/doces } \\
\hline Elevado consumo & 1,00 & & 0,011 & 1,00 & & 0,001 \\
\hline Baixo consumo & 1,78 & $1,14-2,79$ & & 1,08 & $1,03-1,14$ & \\
\hline
\end{tabular}

* Considerou-se o quintil superior da distribuição de escores de cada padrão para elevado consumo dos padrões alimentares. 
A Tabela 3 também mostra que, mesmo após o controle na análise multivariada, mantém-se o efeito protetor do baixo consumo de PA-vegetais para a obesidade geral e do baixo consumo de PA-nozes/oleaginosas sobre a obesidade abdominal. Embora esses resultados pareçam inconsistentes com a literatura científica, após análi- ses estratificadas por idade, escolaridade e classe econômica, verifica-se a presença de efeito de modificação por essas variáveis nas associações desses dois padrões alimentares com obesidade geral e abdominal.

$\mathrm{Na}$ análise estratificada, o baixo consumo de PA-vegetais conservou o efeito protetor para

Análise multivariada para obesidade geral (índice de massa corporal - IMC) e abdominal (circunferência da cintura - CC) incluindo variáveis demográficas, sócio-econômicas e elevado consumo dos padrões alimentares * em mulheres adultas de São Leopoldo, Rio Grande do Sul, Brasil, 2003 ( $N=1.026$ ).

\begin{tabular}{|c|c|c|c|c|c|c|}
\hline \multirow[t]{2}{*}{ Variáveis } & \multicolumn{3}{|c|}{ IMC } & \multicolumn{3}{|c|}{$\mathrm{CC}$} \\
\hline & RP & IC95\% & Valor de $p$ & RP & IC95\% & Valor de $p$ \\
\hline \multicolumn{7}{|l|}{ Sócio-demográficas ** } \\
\hline \multicolumn{7}{|l|}{ Classe econômica } \\
\hline "D+E" & 1,00 & & 0,372 & 1,00 & & 0,456 \\
\hline "C" & 0,88 & $0,64-1,22$ & & 0,96 & $0,91-1,02$ & \\
\hline "A+B" & 0,74 & $0,49-1,12$ & & 0,98 & $0,92-1,05$ & \\
\hline \multicolumn{7}{|l|}{ Escolaridade (anos) } \\
\hline $0-5$ & 1,00 & & 0,028 & 1,00 & & 0,001 \\
\hline $6-10$ & 0,77 & $0,55-1,07$ & & 0,91 & $0,86-0,96$ & \\
\hline 11 ou mais & 0,57 & $0,38-0,87$ & & 0,89 & $0,84-0,94$ & \\
\hline \multicolumn{7}{|l|}{ Trabalho } \\
\hline Não & 1,00 & & 0,055 & 1,00 & & 0,001 \\
\hline Sim & 0,76 & $0,58-1,00$ & & 0,92 & $0,88-0,96$ & \\
\hline \multicolumn{7}{|l|}{ Idade (anos) } \\
\hline $20-35$ & 1,00 & & $<0,001$ & 1,00 & & $<0,001$ \\
\hline $36-60$ & 1,88 & $1,36-2,60$ & & 1,11 & $1,06-1,15$ & \\
\hline \multicolumn{7}{|l|}{ Estado civil } \\
\hline Com companheiro & 1,00 & & 0,228 & 1,00 & & 0,176 \\
\hline Sem companheiro & 0,83 & $0,61-1,12$ & & 0,96 & $0,92-1,01$ & \\
\hline \multicolumn{7}{|l|}{ 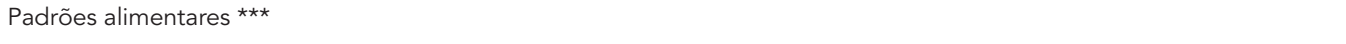 } \\
\hline \multicolumn{7}{|l|}{ PA-vegetais } \\
\hline Elevado consumo & 1,00 & & 0,004 & $\#$ & & - \\
\hline Baixo consumo & 0,64 & $0,47-0,86$ & & & & \\
\hline \multicolumn{7}{|l|}{ PA-frutas } \\
\hline Elevado consumo & 1,00 & & 0,001 & 1,00 & & 0,070 \\
\hline Baixo consumo & 2,18 & $1,35-3,53$ & & 1,05 & $0,99-1,11$ & \\
\hline \multicolumn{7}{|l|}{ PA-nozes/oleaginosas } \\
\hline Elevado consumo & \# & & & 1,00 & & 0,088 \\
\hline Baixo consumo & & & & 0,93 & $0,89-0,98$ & \\
\hline \multicolumn{7}{|c|}{ PA-pão/aipim/batata doce } \\
\hline Elevado consumo & \# & & & 1,00 & & 0,460 \\
\hline Baixo consumo & & & & 0,98 & $0,92-1,03$ & \\
\hline \multicolumn{7}{|l|}{ PA-chocolate/doces } \\
\hline Elevado consumo & 1,00 & & 0,241 & 1,00 & & 0,323 \\
\hline Baixo consumo & 1,30 & $0,83-2,05$ & & 1,03 & $0,97-1,09$ & \\
\hline
\end{tabular}

* Foi considerado elevado consumo o quintil superior;

** Ajustadas por variáveis do mesmo nível;

*** Ajustadas por variáveis do mesmo nível e pelas variáveis do nível superior com $p \leq 0,1$;

\# Este padrão alimentar apresentou $\mathrm{p}>0,2$ na análise bivariada com IMC. 
obesidade geral apenas para as mulheres com baixa escolaridade $(\mathrm{RP}=0,55$; IC95\%: 0,38-0,88; $\mathrm{p}=0,003)$, baixa classe econômica " $\mathrm{D}+\mathrm{E}$ " (RP = 0,58; IC95\%: 0,33-1,01; $\mathrm{p}=0,054)$ e com idade acima de 35 anos (RP = 0,68; IC95\%: 0,49-0,95; $\mathrm{p}=$ 0,025), desaparecendo a proteção nas mulheres com maior escolaridade, melhor classe econômica e aquelas mais jovens. Para o baixo consumo de PA-nozes/oleaginosas, a proteção para obesidade abdominal manteve-se apenas para as mulheres do tercil superior de escolaridade $(\mathrm{RP}=0,91$; IC95\%: 0,85-0,98; $\mathrm{p}=0,024)$, classes econômicas "A+B" (RP = 0,91; IC95\%: 0,85-0,97; $\mathrm{p}=0,008)$ e "C" (RP = 0,90; IC95\%: 0,82-0,99; $\mathrm{p}=$ $0,038)$, e aquelas mais jovens $(\mathrm{RP}=0,90$; IC95\%: $0,82-1,00 ; p=0,057)$, desaparecendo para as mulheres com menor escolaridade, baixa classe econômica e aquelas mulheres categorizadas no grupo etário com mais idade (dados não apresentados em tabelas).

Salienta-se que, na análise estratificada, baixo consumo do PA-frutas associou-se com risco de obesidade geral entre as mulheres com maior escolaridade (RP = 2,54; IC95\%: 1,22-5,26; $\mathrm{p}=$ 0,012 ), categorizadas no grupo etário com mais idade (RP = 2,45; IC95\%: 1,48-4,07; p < 0,001) e pertencentes às classes econômicas mais elevadas $\left(\mathrm{RP}_{\text {“A+B" }}=1,93\right.$; IC95\%: 1,02-3,63; $\mathrm{p}=0,041 \mathrm{e}$ $\mathrm{RP}_{\text {“ }} \mathrm{C}$ " = 4,02; IC95\%: 1,28-12,65; $\mathrm{p}=0$ 0,017), além de ter sido identificado como fator de risco para obesidade abdominal entre as mulheres com maior escolaridade ( $\mathrm{RP}=1,10$; IC95\%: 1,03-1,17; $p=0,006)$. Efeito de risco limítrofe para obesidade abdominal foi observado para as mulheres pertencentes à classe "C" e mais velhas com baixo consumo desse padrão alimentar (dados não apresentados em tabelas).

\section{Discussão}

O presente estudo transversal, de base populacional, analisou a associação de cinco padrões alimentares com obesidade geral e abdominal, entre mulheres adultas. Encontrou-se menor prevalência de excesso de peso em relação a dois estudos realizados em outro município na Região Sul do Brasil, tanto pelo indicador de IMC (49,3\% vs. 53,1\%) ${ }^{4}$ como pela circunferência da cintura $(46,2 \%$ vs. $61,9 \%) 5$. Destaca-se que, embora a obesidade abdominal no nível I encontrada em nosso estudo seja a mesma (23\%) daquela no estudo de referência 5 , houve elevada diferença nas prevalências de obesidade abdominal nível II $(23,3 \%$ vs. $38,7 \%)$.

Após as análises ajustadas, apenas os padrões alimentares considerados saudáveis apresentaram associação com os indicadores de obesida- de. O PA-vegetais e o PA-frutas estiveram associados à obesidade geral, entretanto com efeitos contrários. O baixo consumo de PA-frutas mostrou-se como fator de risco para obesidade geral, enquanto o baixo consumo de PA-vegetais teve efeito protetor, mesmo após controlar para idade, estado civil, escolaridade, situação de emprego e classe econômica na análise ajustada. Já o baixo consumo do PA-nozes/oleaginosas mostrou efeito protetor para a obesidade abdominal.

A complexidade das relações entre padrão alimentar e obesidade ficou ainda mais evidenciada quando se consideraram as análises estratificadas. Por meio delas foi possível perceber a modificação de efeito pela escolaridade, classe econômica e idade na associação do baixo consumo de PA-frutas e PA-vegetais com obesidade. Por um lado, o baixo consumo do PA-vegetais conservou o efeito protetor para obesidade geral apenas para as mulheres pertencentes ao tercil inferior de escolaridade, baixas classes econômicas ("D+E") e também para mulheres com idade acima de 35 anos, desaparecendo a proteção nas mulheres com maior escolaridade, melhor classe econômica e naquelas mais jovens. Parte da explicação destes achados pode ser pela presença de fator de confusão residual, por exemplo, em mulheres acima de 35 anos, há uma série de outros fatores que determinam a obesidade, inclusive hormonais, mas que não foram medidos neste estudo e, conseqüentemente, não foram controlados na análise. Já a relação direta de PAvegetais com obesidade, em mulheres de piores condições sócio-econômicas, pode expressar três situações. Mulheres de baixa renda, entre as quais a obesidade é mais prevalente, têm consciência de que deveriam consumir padrões mais saudáveis e então superestimam a ingestão de vegetais na sua dieta. Achados no Sul do Brasil com mães de baixa renda evidenciaram a super estimativa de ingestão calórica para seus filhos desnutridos 36. Outra possível explicação seria que o baixo consumo de vegetais estaria associado também ao baixo consumo de todos os alimentos entre as mulheres com piores condições sócio-econômicas e, assim, a baixa ingestão calórica seria a causa da proteção observada. Todavia, como o instrumento só avaliava freqüência de ingestão dos alimentos (QFA), não foi possível realizar ajuste para calorias totais da dieta. E, por fim, a informação sobre o consumo de PA-vegetais seria verdadeira, evidenciando a causalidade reversa.

Por outro lado, ao se estratificar a amostra em tercis de escolaridade e por idade $(\leq 35$ anos e $>$ 35 anos), observou-se que o risco de obesidade, tanto geral como abdominal, com o baixo consumo de PA-frutas, manteve-se elevado apenas 
para as mulheres do tercil superior de escolaridade e para as mulheres categorizadas no grupo etário com mais idade. Esse achado, certamente, evidencia um marcador de comportamento de risco nesse grupo específico de mulheres, uma vez que esse desfecho é fortemente relacionado com o estilo de vida.

O delineamento transversal, utilizado no presente estudo, em algumas circunstâncias poderia ser considerado inadequado para investigação de padrões alimentares e características antropométricas, especialmente, pela potencial causalidade reversa nessa relação e também pelo viés de informação 30,37 . Na associação em estudo, a causalidade reversa poderia ocorrer quando pessoas com excesso de peso estariam alimentando-se de forma mais saudável do que o usual, no momento da entrevista. No presente estudo, utilizou-se um QFA sobre o consumo no último mês. Este intervalo de tempo poderia ser mais sensível ao efeito da causalidade reversa do que a avaliação de um período mais longo, como por exemplo, alimentação no último ano. Efetuou-se a análise dos dados sem as mulheres que referiram estar fazendo dieta $(n=120)$ e não foram observadas diferenças nos achados. O viés de informação, também, poderia ser proveniente do sub ou do super-relato de alimentos da dieta ou, ainda, do erro nas medidas antropométricas. Para reduzir a possibilidade de erro sistemático nas informações de peso, estatura e circunferência da cintura, que originaram os desfechos, foram realizados intensos treinamentos com as entrevistadoras, tendo-se o cuidado para que duas medidas independentes fossem coletadas durante o trabalho de campo, com posterior comparação dessas. O subrelato de alimentos calóricos e o super-relato de alimentos saudáveis têm sido observados na literatura, particularmente por pessoas com excesso de peso $37,38,39$, e nossos resultados devem ser interpretados sob essa possibilidade, por tratar-se de um viés de difícil controle na coleta de dados e sem controle na análise.

De um modo geral, a associação de padrões alimentares com IMC ou com obesidade em mulheres tem sido menos evidente do que entre os homens ${ }^{30}$. Cálculos a posteriori demonstraram que o tamanho da amostra deste estudo não apresentou poder estatístico suficiente para evidenciar efeitos de risco ou proteção menores do que $50 \%$, tanto para as associações do PA-vegetais como para o PA-pão/aipim/batata doce sobre a obesidade geral. Entretanto, para os outros três padrões alimentares, a amostra de 1.026 mulheres foi capaz de identificar como significativa uma medida de efeito maior ou igual a 1,5 , com poder de $80 \%$, $\alpha$ de 0,05 e razão de não expostos:expostos de 4:1 (quintis inferiores:quintil superior de consumo) para ambos os desfechos (obesidade geral e abdominal).

Salienta-se que, no presente estudo, vegetais e frutas geraram dois padrões alimentares diferentes. Na comparação com outros estudos, nota-se que frutas e vegetais geralmente constituem um mesmo padrão alimentar. Outro aspecto que merece ser ressaltado refere-se à falta de consistência da associação dos padrões alimentares, obtidos pelos métodos a priori (índices de dieta) ou a posteriori (análise fatorial ou análise de agrupamento), com obesidade ou índice de massa corporal apontada em recente revisão ${ }^{30}$. Alguns estudos verificaram associação inversa do elevado consumo de padrões saudáveis com excesso de peso 40,41,42 (como o consumo do nosso PA-frutas), outro, associação positiva 24 (resultado consistente ao consumo do PA-vegetais e do PA-nozes/oleaginosas deste estudo) e em outro ainda, essa associação não é encontrada 43 , como nos demais padrões identificados no presente estudo.

Ao investigar mudança de peso, o estudo de acompanhamento de Schulze et al. ${ }^{40}$, com 51.670 mulheres, identificou que o aumento do consumo do padrão "prudente" - que corresponderia aos alimentos presentes nos PAvegetais, PA-frutas e PA-nozes/oleaginosas do presente estudo - estaria associado ao menor ganho de peso.

No estudo de Greenwood et al. 41, com uma amostra de 33.971 mulheres com 35-69 anos pertencentes ao UK Women's Cohort Study, foram obtidos sete padrões alimentares. Entre esses, dois padrões vegetarianos e o padrão health conscious (com elevado consumo de cereais, alimentos integrais, iogurte, peixe, frutas, verduras e produtos lácteos desnatados) foram consumidos, em geral, por mulheres com maior escolaridade, que fumavam menos e pertencentes aos grupos sócio-econômicos mais elevados. Além disso, os autores observaram que as mulheres com padrões de consumo alimentar mais saudáveis, principalmente os padrões vegetarianos, apresentavam maiores níveis de atividade física e tinham menor IMC.

Os resultados de outro estudo de coorte com 1.265 pessoas, de 36 a 53 anos, também sugerem uma associação inversa do padrão "frutas, vegetais e produtos lácteos” com IMC $(\mathrm{p}=0,004)$ e com CC ( $p<0,001)$, em mulheres 42 .

Contudo, no Brasil, o padrão alimentar "misto" selecionado por Sichieri et al. 24 , que inclui verduras, legumes, frutas, carne, frango, peixe, produtos lácteos, esteve associado de maneira positiva ao IMC, enquanto um estudo transversal com amostra do Sul do país, também utilizando 
análise fatorial, identificou um padrão alimentar composto de frutas e vegetais que não revelou associação com excesso de peso 43 .

Já no estudo de coorte realizado por Tseng \& DeVellis ${ }^{4}$ com 5.794 participantes, de 20 a 74 anos de idade, não se verificou associação dos dois padrões identificados - "frutas e vegetais" e "amido e carne vermelha" - com obesidade geral ou abdominal. Observou-se, no entanto, que os fatores associados com o alto consumo do padrão "frutas e vegetais" foram os mesmos associados ao baixo consumo do padrão "amido e carne vermelha". Entre tais fatores estavam maior idade e escolaridade, sexo feminino, morar na zona urbana, uso de suplemento, atividade física freqüente e a tentativa de emagrecimento, evidenciando a causalidade reversa. $\mathrm{O}$ padrão "Espanhol-Mediterrâneo", identificado na amostra com 3.847 adultos do estudo de Sánchez-Villegas et al. 45 , foi constituído por vegetais, peixes, frutas, óleo de oliva, legumes, batatas, nozes, castanhas e aves e consumido por pessoas mais ativas fisicamente e com história prévia de obesidade.

Nas últimas décadas diversos estudos têm utilizado padrões alimentares para estabelecer a relação da dieta com doenças crônicas não transmissíveis 46,47. Essa abordagem surgiu como uma alternativa, visto que as pessoas não consomem nutrientes nem alimentos de forma isolada e, portanto, os padrões alimentares representariam melhor a complexa exposição que é a alimentação. Destaca-se, porém, que no processo de identificação dos padrões alimentares, derivados a posteriori, o investigador toma decisões que podem afetar o número e o tipo de padrões 48,49 . Logo, há necessidade de conhecer melhor a validade e a confiabilidade dos padrões alimentares e sua capacidade de predizer doenças com longo período de risco em populações 19,48.

Com base nos resultados e na literatura existente, sugere-se a otimização dos desenhos de estudos epidemiológicos para a investigação de dieta e doenças crônicas. Conforme $\mathrm{Hu}$ 19, padrões alimentares não seriam uma decisão adequada para investigar um efeito causado por um nutriente específico devido à diluição do efeito. Ao mesmo tempo, considerando-se a eficiência (custo/benefício) das investigações científicas, os estudos com desenhos longitudinais que utilizam amostras grandes, superiores a $10 \mathrm{mil}$ pessoas 40,50 , poderiam focar suas análises nas exposições de longos períodos, que envolvem complexas interações entre os nutrientes sobre as doenças crônicas não transmissíveis. Por outro lado, não menos importante, seriam os delineamentos transversais de base populacional, com amostras representativas, para o estudo do efeito dos padrões alimentares, pois além de caracterizarem-se em alternativas rápidas e baratas, comparados com os estudos de coorte, são fundamentais para monitorar e subsidiar ações educativas para promoção de hábito alimentar saudável na população de referência ou em outras populações com características similares. 


\section{Resumo}

Com objetivo de estudar a associação de padrões alimentares com obesidade, realizou-se estudo transversal de base populacional com amostra representativa de 1.026 mulheres (20 a 60 anos) em São Leopoldo, Rio Grande do Sul, Brasil. A obesidade geral foi avaliada pela utilização de índice de massa corporal (IMC $\geq$ $30 \mathrm{~kg} / \mathrm{m}^{2}$ ) e a adiposidade abdominal, circunferência da cintura (CC $\geq 88 \mathrm{~cm})$. Os padrões alimentares foram identificados por análise fatorial. Para análise multivariada, foi utilizada regressão de Poisson. Entre o total de mulheres, 18\% (IC95\%: 15,66-20,53) tinham obesidade geral e 23,3\% (IC95\%: 20,72-26,06) abdominal. Após controle para fatores de confusão, o baixo consumo do PA-frutas associou-se positivamente com o IMC (RP = 2,18; IC95\%: 1,35-3,53; $p=0,001)$. Já o baixo consumo do PA-vegetais apresentou efeito protetor para o aumento nos níveis de IMC $(R P=0,64$; IC95\%: $0,47-0,86 ; p=0,004)$ e o do PA-nozes/oleaginosas para o aumento na medida da CC (RP = 0,93; IC95\%: 0,89$0,98 ; p=0,008)$. O estudo aponta para a complexidade envolvida na relação entre padrões alimentares e obesidade e a necessidade de novos estudos, objetivando o melhor entendimento do tema.

Comportamento Alimentar; Obesidade; Gordura Abdominal; Mulheres

\section{Referências}

1. World Health Organization. Obesity: preventing and managing the global epidemic. Report of a WHO consultation on obesity. Geneva: World Wealth Organization; 2000.

2. Instituto Brasileiro de Geografia e Estatística. Estudo Nacional da Despesa Familiar - ENDEF: dados preliminares, consumo alimentar, antropometria. v. 1. Rio de Janeiro: Instituto Brasileiro de Geografia e Estatística; 1977.

3. Instituto Brasileiro de Geografia e Estatística. Pesquisa de orçamentos familiares 2002-2003: análise da disponibilidade domiciliar de alimentos e do estado nutricional no Brasil. Rio de Janeiro: Instituto Brasileiro de Geografia e Estatística; 2004.

4. Gigante DP, Dias-da-Costa JS, Olinto MTA, Menezes AMB, Macedo S. Obesidade da população adulta de Pelotas, Rio Grande do Sul, Brasil e associação com nível sócio-econômico. Cad Saúde Pública 2006; 22:1873-9.

5. Olinto MTA, Nácul LC, Dias-da-Costa JS, Gigante DP, Menezes AMB, Macedo S. Níveis de intervenção para obesidade abdominal: prevalência e fatores associados. Cad Saúde Pública 2006; 22:1207-15.

\section{Colaboradores}

G. Perozzo, J. Sarriera e M. P. Pattussi trabalharam na análise dos dados e na redação do artigo. M. T. A. Olinto e J. S. Dias-da-Costa são os coordenadores do projeto e estiveram envolvidos em todas suas etapas, desde a elaboração do projeto até a análise dos dados e redação do artigo. R. L. Henn colaborou na discussão dos resultados.
6. Brewer EA, Kolotkin RL, Baird DD. The relationship between eating behaviors and obesity in African American and Caucasian women. Eat Behav 2003; 4:159-71.

7. Ma Y, Bertone ER, Stanek EJ, Reed GW, Herbert JR, Cohen NL, et al. Association between eating patterns and obesity in a free-living US adult population. Am J Epidemiol 2003; 158:85-92.

8. McCrory MA, Suen VM, Roberts SB. Biobehavioral influences on energy intake and adult weight gain. J Nutr 2002; 132:3830S-4S.

9. Bell EA, Castellanos VH, Pelkman CL, Thorwart ML, Rolls BJ. Energy density of foods affects energy intake in normal-weight women. Am J Clin Nutr 1998; 67:412-20.

10. Bray GA, Popkin BM. Dietary fat intake does affect obesity! Am J Clin Nutr 1998; 68:1157-73.

11. Stubbs RJ, Mazlan N, Whybrow S. Carbohydrates, appetite and feeding behavior in humans. J Nutr 2001; 131:2775S-81S.

12. Willett WC. Dietary fat and obesity: an unconvincing relation. Am J Clin Nutr 1998; 68:1149-50. 
13. Willett WC, Leibel RL. Dietary fat is not a major determinant of body fat. Am J Med 2002; 113 Suppl 9B:47S-59S.

14. Halkjaer J, Tjønneland A, Thomsen BL, Overvad K, Sørensen TI. Intake of macronutrients as predictors of 5-y changes in waist circumference. Am J Clin Nutr 2006; 84:789-97.

15. Jenkins DJ, Kendall CW, Augustin LS, Franceschi S, Hamidi M, Marchie A, et al. Glycemic index: overview of implications in health and disease. Am J Clin Nutr 2002; 76:266S-73S.

16. Koh-Banerjee P, Chu NF, Spiegelman D, Rosner B, Colditz G, Willett WC, et al. Prospective study of the association of changes in dietary intake, physical activity, alcohol consumption, and smoking with 9-y gain in waist circumference among 16,587 US men. Am J Clin Nutr 2003; 78:719-27.

17. Newby PK, Muller D, Hallfrisch J, Qiao N, Andres R, Tucker KL. Dietary patterns and changes in body mass index and waist circumference in adults. Am J Clin Nutr 2003; 77:1417-25.

18. Halkjaer J, Sørensen TI, Tjønneland A, Togo P, Holst C, Heitmann BL. Food and drinking patterns as predictors of 6-year BMI-adjusted changes in waist circumference. Br J Nutr 2004; 92:735-48.

19. Hu FB. Dietary pattern analysis: a new direction in nutritional epidemiology. Curr Opin Lipidol 2002; 13:3-9.

20. Lin H, Bermudez OI, Tucker KL. Dietary patterns of Hispanic elders are associated with acculturation and obesity. J Nutr 2003; 133:3651-7.

21. Maskarinec G, Novotny R, Tasaki K. Dietary patterns are associated with body mass index in multiethnic women. J Nutr 2000; 130:3068-72.

22. Newby PK, Muller D, Hallfrisch J, Andres R, Tucker KL. Food patterns measured by factor analysis and anthropometric changes in adults. Am J Clin Nutr 2004; 80:504-13.

23. Sichieri R. Dietary patterns and their associations with obesity in the Brazilian city of Rio de Janeiro. Obes Res 2002; 10:42-8.

24. Sichieri R, Castro JFG, Moura AS. Fatores associados ao padrão de consumo alimentar da população brasileira urbana. Cad Saúde Pública 2003; 19 Supp 1:S47-53.

25. Wirfält E, Hedblad B, Gullberg B, Mattisson I, Andrén C, Rosander U, et al. Food patterns and components of the metabolic syndrome in men and women: a cross-sectional study within the Malmö Diet and Cancer cohort. Am J Epidemiol 2001; 154:1150-9.

26. Wirfält E, Mattisson I, Gullberg B, Berglund G. Food patterns defined by cluster analysis and their utility as dietary exposure variables: a report from the Malmö Diet and Cancer Study. Public Health Nutr 2000; 3:159-73.

27. Fung TT, Rimm EB, Spiegelman D, Rifai N, Tofler GH, Willett WC, et al. Association between dietary patterns and plasma biomarkers of obesity and cardiovascular disease risk. Am J Clin Nutr 2001; 73:61-7.

28. Quatromoni PA, Copenhafer DL, D'Agostino RB, Millen BE. Dietary patterns predict the development of overweight in women: The Framingham Nutrition Studies. J Am Diet Assoc 2002; 102: 1239-46.
29. Togo P, Osler M, Sørensen TI, Heitmann BL. A longitudinal study of food intake patterns and obesity in adult Danish men and women. Int J Obes Relat Metab Disord 2004; 28:583-93.

30. Togo P, Osler M, Sørensen TI, Heitmann BL. Food intake patterns and body mass index in observational studies. Int J Obes Relat Metab Disord 2001; 25:1741-51.

31. Alves ALS, Olinto MTA, Balbinotti MAA, Dias-daCosta JS, Bairros FS. Padrões alimentares de mulheres adultas residentes em área urbana no Sul do Brasil. Rev Saúde Pública 2006; 40:865-73.

32. Masson CR, Dias-da-Costa JS, Olinto MTA, Meneghel S, Costa CC, Bairros F, et al. Prevalência de sedentarismo nas mulheres adultas da cidade de São Leopoldo, Rio Grande do Sul, Brasil. Cad Saúde Pública 2005; 21:1685-94.

33. Associação Brasileira de Empresas de Pesquisa. Códigos e guias: CCEB - Critério de Classificação Econômica Brasil. http://www.abep.org (acessado em 15/Jun/2006).

34. Lean ME, Han TS, Morrison CE. Waist circumference as a measure for indicating need for weight management. BMJ 1995; 311:158-61.

35. Victora CG, Hutty SR, Fuchs SC, Olinto MTA. The role of conceptual frameworks in epidemiological analysis: A hierarchical approach. Int J Epidemiol 1997; 26:224-7.

36. Olinto MT, Victora CG, Barros FC, Gigante DP. Twenty-four-hour recall overestimates the dietary intake of malnourished children. J Nutr 1995; 125:880-4

37. Heitmann BL, Lissner L. Dietary underreporting by obese individuals - is it specific or non-specific? BMJ 1995; 311:986-9.

38. Lissner L, Heitmann BL, Bengtsson C. Population studies of diet and obesity. Br J Nutr 2000; 83 Suppl 1:S21-4.

39. Pomerleau J, Ostbye T, Bright-See E. Potential underreporting of energy intake in the Ontario Health Survey and its relationship with nutrient and food intakes. Eur J Epidemiol 1999; 15:553-7.

40. Schulze MB, Fung TT, Manson JE, Willett WC, Hu FB. Dietary patterns and changes in body weight in women. Obesity (Silver Spring) 2006; 14:1444-53.

41. Greenwood DC, Cade JE, Draper A, Barrett JH, Calvert C, Greenhalgh A. Seven unique food consumption patterns identified among women in the UK Women's Cohort Study. Eur J Clin Nutr 2000; 54:314-20

42. McNaughton SA, Mishra GD, Stephen AM, Wadsworth ME. Dietary patterns throughout adult life are associated with body mass index, waist circumference, blood pressure, and red cell folate. J Nutr 2007; 137:99-105.

43. Henn RL. Padrão alimentar e excesso de peso em uma população adulta da cidade de Porto Alegre, RS, 2005 [Tese de Doutorado]. Porto Alegre: Universidade Federal do Rio Grande do Sul; 2005.

44. Tseng M, DeVellis RF. Fundamental dietary patterns and their correlates among US whites. J Am Diet Assoc 2001; 101:929-32. 
45. Sánchez-Villegas A, Delgado-Rodríguez M, Martínez-González MA, Irala-Estévez J; Seguimiento Universidad de Navarra Group. Gender, age, socio-demographic and lifestyle factors associated with major dietary patterns in the Spanish Project SUN (Seguimiento Universidad de Navarra). Eur J Clin Nutr 2003; 57:285-92.

46. Kant AK. Dietary patterns and health outcomes. J Am Diet Assoc 2004; 104:615-35.

47. Newby PK, Tucker KL. Empirically derived eating patterns using factor or cluster analysis: a review. Nutr Rev 2004; 62:177-203.

48. Moeller SM, Reedy J, Millen AE, Dixon LB, Newby PK, Tucker KL, et al. Dietary patterns: challenges and opportunities in dietary patterns research. J Am Diet Assoc 2007; 107:1233-9.
49. Marchioni DML, Latorre MRDO, Eluf-Neto J, Wünsch-Filho V, Fisberg RM. Identification of dietary patterns using factor analysis in an epidemiological study in São Paulo. São Paulo Med J 2005; 123:124-7.

50. Newby PK, Weismayer C, Akesson A, Tucker KL, Wolk A. Longitudinal changes in food patterns predict changes in weight an body mass index and the effects are greatest in obese women. J Nutr 2006; 136:2580-7.

Recebido em 08/Ago/2007

Versão final reapresentada em 07/Mar/2008

Aprovado em 13/Mar/2008 